QUARTERLY OF APPLIED MATHEMATICS

VOLUME LXIX, NUMBER 1

MARCH 2011, PAGES 105-122

S 0033-569X(2010)01205-0

Article electronically published on December 29, 2010

\title{
WAITING TIME PHENOMENA FORCED BY CRITICAL BOUNDARY CONDITIONS IN CLASSICAL DIFFUSION PROBLEMS
}

\author{
BY
}

\begin{abstract}
A. FASANO (Dip. di Matematica "U.Dini" - Universitá degli Studi di Firenze, viale Morgagni, 67a 50134 Firenze, Italy),

A. MANCINI (Dip. di Matematica "U.Dini" - Universitá degli Studi di Firenze, viale Morgagni, 67a - 50134 Firenze, Italy),

M. PRIMICERIO (Dip. di Matematica "U.Dini" - Universitá degli Studi di Firenze, viale Morgagni, $67 a-50134$ Firenze, Italy),

AND

B. ZALTZMAN (DSEEP, Blaustein Institute for Desert Research, Ben-Gurion University of the Negev, Sede-Boker Campus, 84990, Israel)
\end{abstract}

\begin{abstract}
This paper revisits some very classical initial-boundary value problems for parabolic equations, providing simple examples in which the occurrence of flux discontinuities at the boundary when the unknown function reaches some critical value may give rise to a waiting time phenomenon. A physical interpretation could be a modification of the surface of the considered body taking place at the mentioned critical value, affecting the way the body interacts with the surroundings. The waiting time, whose length (finite or infinite) is a priori unknown allows the system to evolve gradually through the critical state. Some numerical simulations are also presented.
\end{abstract}

1. Introduction. It is well known that the propagation of a gas in a porous medium, governed by the so-called porous medium equation, may exhibit a phenomenon of waiting time, in the sense that, if the gas is initially confined in a bounded region with a suitable density distribution, a finite time will elapse before any motion of the boundary can be observed [3], [1] .

In that case the waiting time phenomenon (as well as the finite speed of propagation of the invasion front) is related to the degeneracy of the governing differential equation.

Received July 21, 2009.

2000 Mathematics Subject Classification. Primary 35K60.

E-mail address: fasano@math.unifi.it

E-mail address: mancini@math.unifi.it

E-mail address: primicerio@math.unifi.it

E-mail address: boris@bgumail.bgu.ac.il

(C)2010 Brown University 
In this paper we want to deal with a different class of phenomena, in which the governing equation is uniformly parabolic (e.g. the heat equation), or even elliptic, and the origin of the waiting time is to be found in some nonlinear condition imposed on the boundary.

In most cases we will refer to boundary value problems on prescribed domains, but we will also consider free boundary problems. In the latter case the condition producing the waiting time phenomenon may be given either at a known part of the boundary, or on the free boundary itself (see section 6).

A situation leading to the occurrence of a waiting time in a heat conduction problem with phase change has been pointed out in [6]. However, such a phenomenon is by no means necessarily related to phase change and we want to illustrate this fact through several examples.

Generally speaking, the waiting time phenomenon in heat conduction problems occurs when boundary conditions exhibit the following situation:

- the entering heat flux at the boundary has a jump when the boundary temperature crosses a critical value $u_{0}$;

- the jump is negative when the temperature increases through $u_{0}$.

When these circumstances occur, if at some time $t_{0}$ the boundary temperature reaches the critical value $u_{0}$, e.g. from below, then it is forced to stay at this critical value for some time, the waiting time, during which the heat flux passes from the value corresponding to $u_{0}^{-}$to the value corresponding to $u_{0}^{+}$.

We can interpret this waiting time as the time needed by the boundary surface to adjust its thermal properties to the new value compatible with the variation of the boundary temperature.

Since the occurrence of waiting time is a local phenomenon, in the following examples we will refer to one-dimensional model problems generally in the half-space where it will be easy to estimate the waiting time. The analysis can be easily adapted to finite domains.

2. A first example of boundary condition generating waiting time phenomena. We consider the following model problem in normalized variables:

$$
\begin{aligned}
& u_{x x}-u_{\tau}=0, x \in(0,+\infty), \tau>0, \\
& u(x, 0)=\phi(x), x \in(0,+\infty), \\
& u_{x}(0, \tau)=\left\{\begin{array}{ll}
-A, & u<0 \\
-A+B, & u>0
\end{array}, \tau>0,\right.
\end{aligned}
$$

where $A$ and $B$ are positive constants, $\phi$ has at most a polynomial growth as $x \rightarrow \infty$ and we look for solutions that have the same property.

Suppose $\phi$ is continuous for $x \geq 0$ and $\phi(0)<0$ and assume that a first instant $\tau=\tau_{0}>0$ exists such that $u\left(0, \tau_{0}\right)=0$. Classical properties of the heat equation ensure that (see e.g. 9)

(i) $u \in C^{\infty}\left([0,+\infty) \times\left(0, \tau_{0}\right]\right)$,

(ii) $\frac{\partial^{2} u}{\partial x^{2}}-\frac{\partial u}{\partial \tau}=0$ also for $x=0,0<\tau<\tau_{0}$, 
(iii) $\frac{\partial^{2 k} u}{\partial x^{2 k}}-\frac{\partial^{k} u}{\partial \tau^{k}}=0, x \geq 0,0<\tau \leq \tau_{0}, k=1,2, \ldots$,

(iv) $\left.\frac{\partial^{k}}{\partial \tau^{k}} \frac{\partial u}{\partial x}\right|_{x=0}=\left.\frac{\partial^{2 k+1} u}{\partial x^{2 k+1}}\right|_{x=0}=0, \tau \in\left(0, \tau_{0}\right], k=1,2, \ldots$,

(v) $u(x, \tau)$ is analytic w.r.t. $x \in[0,+\infty)$, for any $\tau \in\left(0, \tau_{0}\right]$.

Now, evaluate

$$
v_{s}:=\left.\frac{d^{s}}{d \tau^{s}} u(0, \tau)\right|_{\tau=\tau_{0}}, s=1,2, \ldots
$$

and let $n$ be the first value of $s$ such that $v_{s} \neq 0$ (the existence of $n$ follows from (iii), (iv) and (v)).

By definition of $\tau_{0}$, we necessarily have

$$
v_{n}>0,
$$

because of $(\mathrm{v})$, and of the assumption $\phi(0)<0$.

Consequently, defining $\Phi(x)=u\left(x, \tau_{0}\right)$, we have

$$
\begin{aligned}
& \Phi^{\prime}(0)=-A, \\
& \Phi^{(2 k+1)}(0)=0, k=1,2, \ldots, \\
& \Phi^{(2 k)}(0)=0, k<n, \Phi^{(2 n)}(0)>0 .
\end{aligned}
$$

Hence we can write

$$
u\left(x, \tau_{0}\right)=\Phi(x)=-A x+a x^{2 n}+o\left(x^{2 n}\right), a>0 .
$$

Setting $t=\tau-\tau_{0}$, consider the equation

$$
u_{x x}-u_{t}=0, x>0, t>0,
$$

with initial datum

$$
u(x, 0)=\Phi(x), x>0 .
$$

It is immediately seen that if we impose the boundary condition

$$
u_{x}(0, t)=-A
$$

in a time interval $t \in\left(0, t_{1}\right)$, then for any $t_{1}>0$ there exists an interval $(0, \bar{t})$ such that $u(0, t)>0$ in $(0, \bar{t})$ (remember $a>0)$, and hence condition (2.3) is not fulfilled.

On the other hand, imposing the condition

$$
u_{x}(0, t)=-A+B
$$

in some interval, then the solution of (2.5), (2.6), (2.8) in the same time interval is given by (see [4, [5])

$$
u(x, t)=(-A+B) x+\int_{0}^{+\infty}\left(-B \xi+a \xi^{2 n}+o\left(\xi^{2 n}\right)\right)[\Gamma(x, t ; \xi, 0)+\Gamma(-x, t, \xi, 0)] d \xi
$$

where

$$
\Gamma(x, t ; \xi, \tau)=\frac{1}{2 \sqrt{\pi(t-\tau)}} \exp \left[-\frac{(x-\xi)^{2}}{4(t-\tau)}\right]
$$

is the fundamental solution of the heat equation, and the integral in (2.9) represents the solution of the heat equation with zero flux on $x=0$ and initial value $\Phi(x)=(-A+B) x$. 
Thus we have immediately

$$
u(0, t)=-\frac{2}{\sqrt{\pi}} B \sqrt{t}+O\left(t^{n}\right),
$$

so that $u(0, t)$ is negative in a right neighborhood of $t=0$ (i.e., of $\tau=\tau_{0}$ ), again contradicting (2.3).

Thus neither imposing (2.7), nor (2.8) for $\tau>\tau_{0}$ provides a solution to the given problem.

In other words, we are not allowed to switch instantaneously at $\tau=\tau_{0}$ to the reduced flux $-A+B$, nor to keep the same flux $-A$ for $\tau>\tau_{0}$.

From this elementary example it comes out that the boundary condition (2.3) is not well formulated, but it has to be rewritten as

$$
u_{x}(0, t) \in-A+B H(u),
$$

where $H(u)$ is the Heaviside graph

$$
H(u)= \begin{cases}0, & u<0, \\ {[0,1],} & u=0, \\ 1, & u>0 .\end{cases}
$$

This amounts to solving (2.5), (2.6) with the boundary condition

$$
u(0, t)=0,0<t<t_{w},
$$

where $t_{w}$ is the waiting time (which is one of the unknowns of the problem), i.e. the first time such that

$$
u_{x}\left(0, t_{w}\right)=-A+B .
$$

After $t_{w}$ we switch to (2.8). In other words, the waiting time is the time during which the vertical part of the graph $H(u)$ is entirely covered.

It is a simple exercise to solve (2.5), (2.6), (2.14) and evaluate $u_{x}(0, t)$ as

$$
u_{x}(0, t)=2 \int_{0}^{+\infty} \Phi^{\prime}(\xi) \Gamma(0, t ; \xi, 0) d \xi .
$$

For instance if we truncate (2.4) to $\Phi=-A x+a x^{2 n}$, then we have

$$
u_{x}(0, t)=-A+\frac{2^{2 n} n !}{\sqrt{\pi}} a t^{n-\frac{1}{2}},
$$

which provides the length of the waiting time

$$
t_{w}=\left(\frac{\sqrt{\pi} B}{2^{2 n} a n !}\right)^{\frac{2}{2 n-1}}
$$

Returning to case (2.4), it remains to show that solving the problem for $t>t_{w}$, with condition (2.8), we find $u(0, t)$ positive in a neighborhood $t_{w}<t<\hat{t}$.

But this is immediately verified from the definition of $t_{w}$. Indeed by an argument similar to the one that we used to write (2.4) we find that

$$
u\left(x, t_{w}\right)=(-A+B) x+\alpha x^{2 m+1}+o\left(x^{2 m+1}\right)
$$




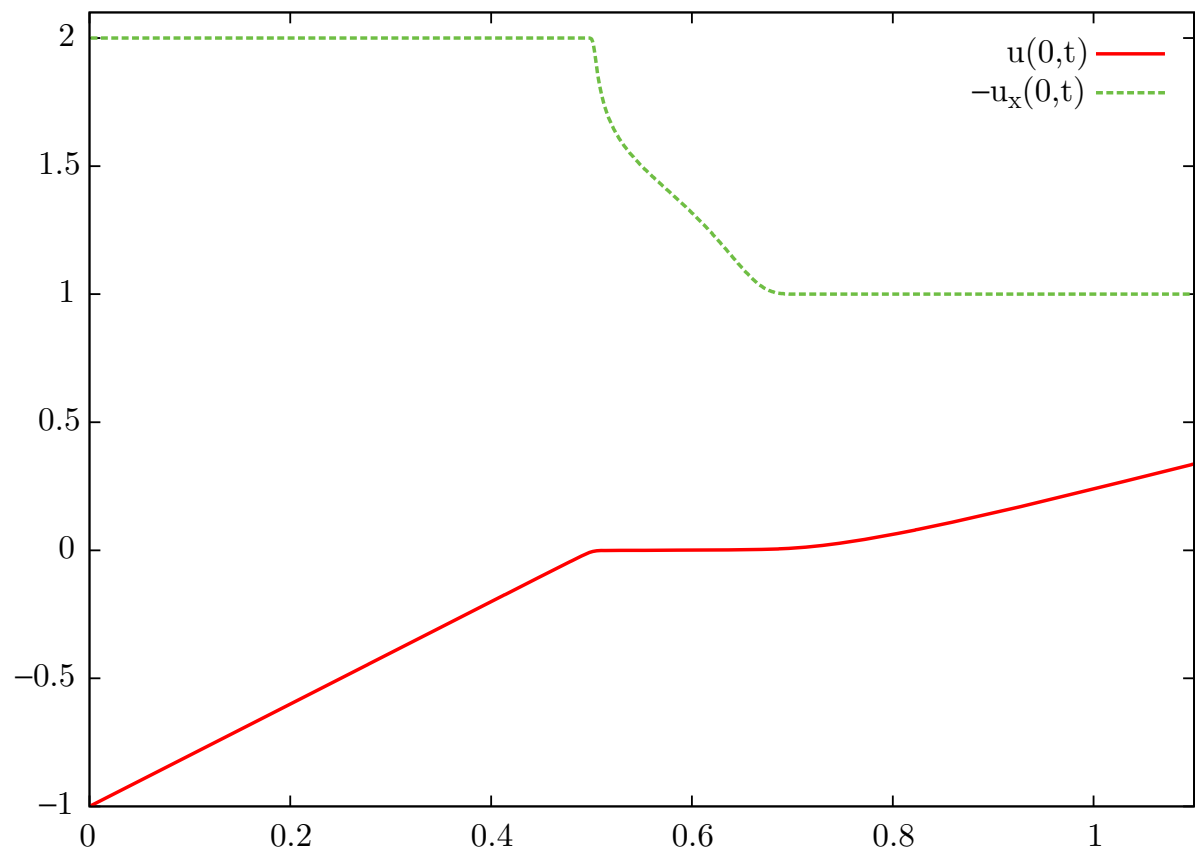

FIG. 1. Waiting time in problem $a$.

for some integer $m \geq 1$ and $\alpha>0$. Therefore

$$
u(x, t)=(-A+B) x+U(x, t),
$$

where $U$ solves a problem with zero flux on $x=0$ and initial datum that is positive in a neighborhood of $x=0$.

In Figure 1 we show $u(0, t)$ (solid line) and $u(1, t)$ (dashed line), found by solving numerically problem $a$ : the heat equation in the slab $x \in(0,1)$ with the following conditions, $u(x, 0)=x^{2}-2 x-1, u_{x}(1, r)=0, u_{x}(0, t) \in-2+H(u(0, t))$.

In Figure 2 we show $u(x, t)$ for fixed $t$ (step 0.2 ) with a zoom close to $x=0, u=0$; in this example the initial condition is $u(x, 0)=-1$ (problem $b$ ).

REMARK 2.1. Of course, cases of infinite waiting time can occur in bounded domains. The same remark also applies to the problem stated in the sequel.

REMARK 2.2. Depending on the initial condition or on the conditions at other boundaries, the waiting time phenomenon may occur more than once. Figure 3 shows a case of multiple occurrence, when $u(1, t)=-\sin (\pi t)$.

3. Robin's law with discontinuous heat exchange. We will see that waiting time may also occur when the boundary condition is of Robin-type as, for instance,

$$
u_{x}(0, t)=h(u(0, t))(u(0, t)-1),
$$




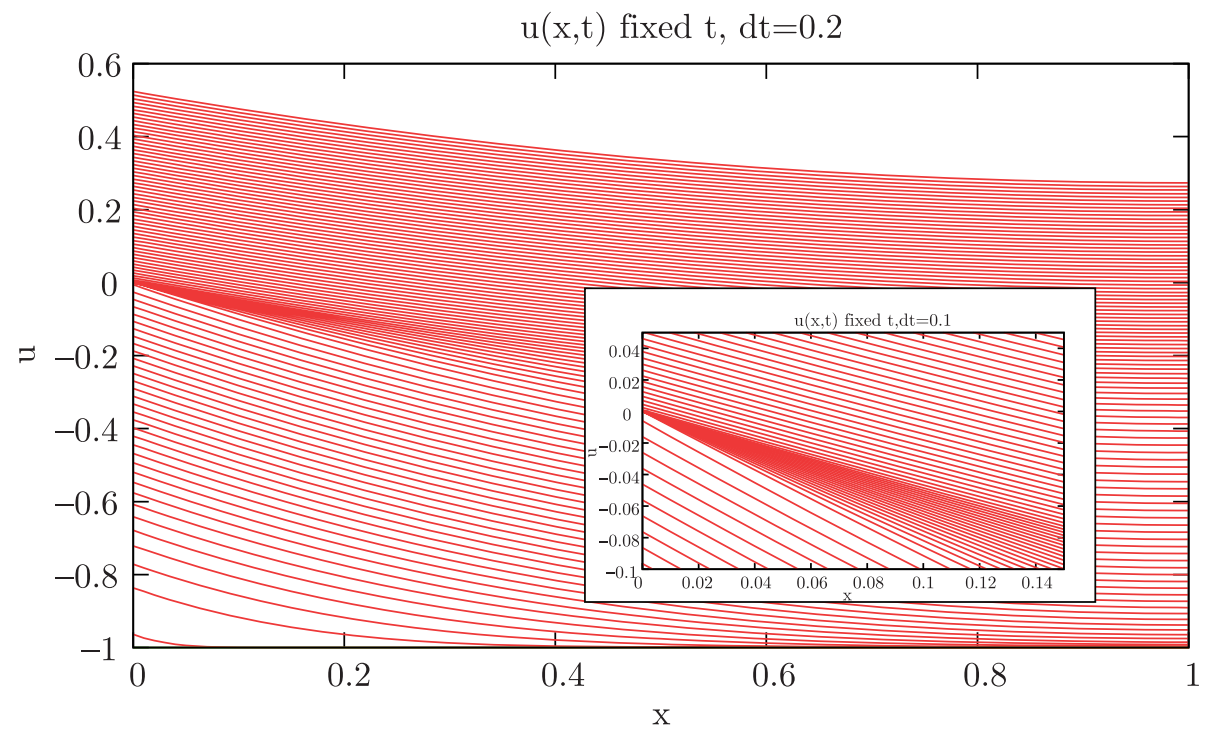

FIG. 2. Waiting time in problem $b$.

and $h$ is a discontinuous function of $u(0, t)$ with a negative jump at some critical value. That is, assume (2.2) with $\phi(0)<0$ and

$$
h= \begin{cases}A, & u(0, t)<0, \\ A-B, & u(0, t)>0,\end{cases}
$$

with $A, B>0, A-B>0$.

The physical interpretation of (3.1), (3.2) can be as follows: the surface $x=0$ changes its heat transmission properties when the temperature $u=0$ is crossed, and consequently the heat exchange coefficient with a thermostat kept at temperature $\bar{u}=1$ is reduced from $A$ to $A-B$.

As in the previous case, we want to show that a waiting time is produced. We still refer to the domain $x>0$. Using the same notation of section 2 we will have to solve (2.5), (2.6) with $\Phi(x)$ given by (2.4). We neglect for simplicity the term $o\left(x^{2 n}\right)$ and we take $n=1$, but the argument is valid in general.

We have to show that for any $\hat{t}>0$, there exists $\tilde{t}>0$ such that:

(i) if we impose

$$
u_{x}(0, t)=A[u(0, t)-1], t \in(0, \hat{t}),
$$

we get $u(0, t)>0$ in $(0, \tilde{t})$;

(ii) if we impose

$$
u_{x}(0, t)=(A-B)[u(0, t)-1], t \in(0, \hat{t}),
$$

then $u(0, t)<0$ in $(0, \tilde{t})$.

Statement (i) is immediately verified, since imposing condition (3.3) (i.e. the same condition holding for $t<0$, which means $\left.\tau<\tau_{0}\right)$ makes $u_{t}(x, t)$ continuous in $(0,0)$ and hence positive and equal to $a$. 


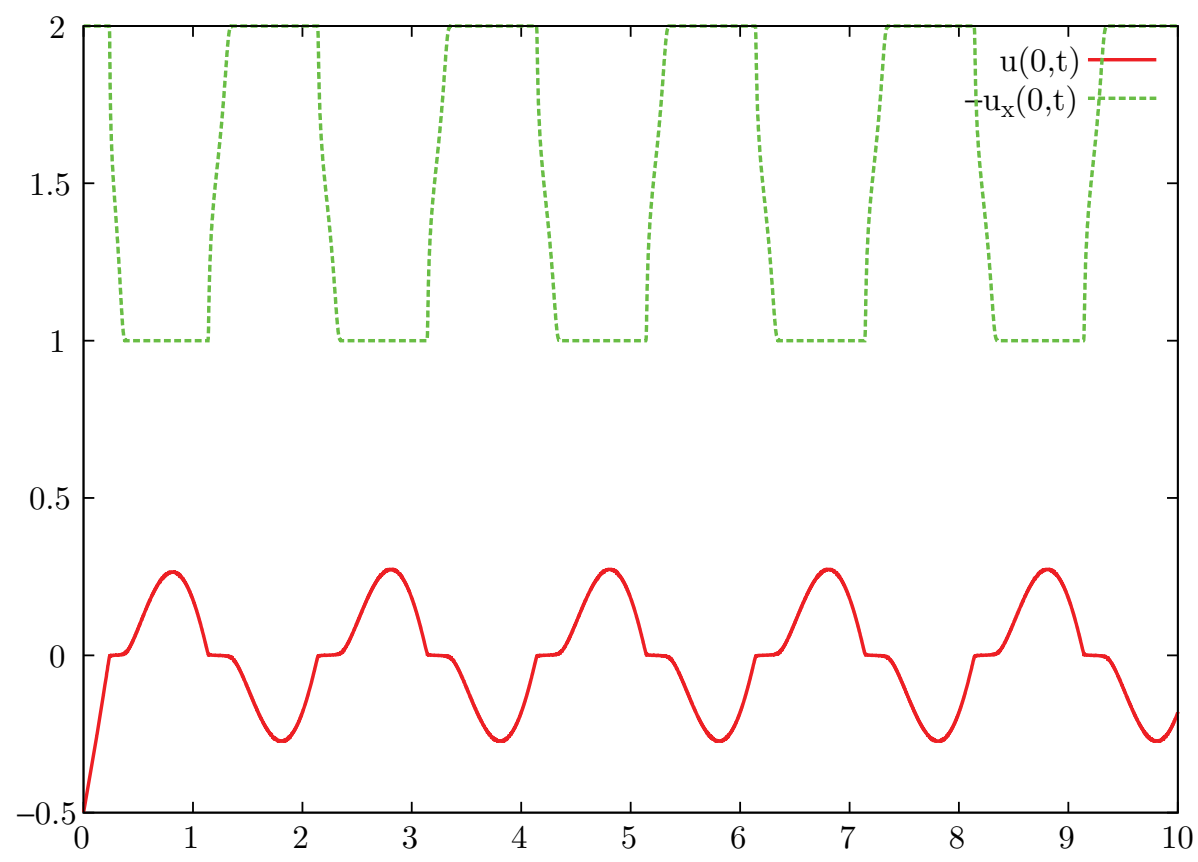

FIG. 3. Multiple occurrence of waiting time; see Remark 2.2

To prove (ii) we have to write the solution of (2.5), (2.6), (3.4), and prove that $u(0, t)<$ 0 in some $(0, \tilde{t})$.

We look for the solution in the form

$$
u(x, t)=\int_{0}^{+\infty} \Phi(\xi) G(x, t ; \xi, 0) d \xi+\int_{0}^{t} \mu(\tau) \Gamma(x, t ; 0, \tau) d \tau
$$

where

$$
G(x, t ; \xi, \tau)=\Gamma(x, t ; \xi, \tau)-\Gamma(-x, t ; \xi, \tau)
$$

is the Green's function for the half-plane $x>0$ and $\mu(t)$ will be chosen so that (3.4) is satisfied.

Since

$$
u(0, t)=\int_{0}^{t} \frac{\mu(\tau)}{2 \sqrt{\pi(t-\tau)}} d \tau, t>0
$$

and

$$
u_{x}(0, t)=\frac{1}{\sqrt{\pi t}} \int_{0}^{\infty} \Phi^{\prime} e^{-\frac{\xi^{2}}{4 t}} d \xi-\frac{1}{2} \mu(t)
$$

(recall the jump relation for the double-layer heat potential; see [8]), we find that $\mu(t)$ has to solve the following Volterra integral equation:

$$
\frac{1}{2} \mu(t)+(A-B) \int_{0}^{t} \frac{\mu(\tau)}{2 \sqrt{\pi(t-\tau)}} d \tau=-B+\frac{2 a}{\sqrt{\pi}} \sqrt{t},
$$

showing that $\mu(t)$ is negative in some neighborhood of $t=0$. Thus (ii) is true. 
Consequently, we will have to state (3.1) in the form

$$
u_{x}(0, t) \in[A-B H(u(0, t))](u(0, t)-1) .
$$

The waiting time is found by solving (2.5), (2.6) with the boundary condition

$$
u(0, t)=0,0<t<t_{w}
$$

and $t_{w}$ is the first time such that

$$
u_{x}\left(0, t_{w}\right)=-A+B
$$

i.e.

$$
t_{w}=\pi \frac{B^{2}}{16 a^{2}}
$$

For $t>t_{w}$ we impose

$$
u_{x}(0, t)=(A-B)[u(0, t)-1] .
$$

If we prove that $u(0, t)>0$ in some neighborhood $\left(t_{w}, t_{w}+\delta\right)$, the solution found by means of the above procedure satisfies (3.10).

Thus, we have to prove that the solution of the heat equation in the region $x>0$, $t^{\prime}>0$ with condition

$$
\begin{aligned}
& u(x, 0)=-D x+\alpha x^{2 n+1}+o\left(x^{2 n+1}\right), x>0, \\
& u_{x}\left(0, t^{\prime}\right)=D\left(u\left(0, t^{\prime}\right)-1\right), t^{\prime}>0
\end{aligned}
$$

is positive in $x=0$ for some interval $t^{\prime} \in(0, \hat{t})$.

In (3.14), (3.15) we have written $t^{\prime}=t-t_{w}$ and $D=A-B, m$ integer, $m \geq 1$ and $\alpha>0$.

But writing

$$
u=-D x+v,
$$

we see that $v$ satisfies the heat equation with

$$
v_{x}=D v \text { for } x=0
$$

and

$$
v(x, 0)=\alpha x^{2 n+1}+o\left(x^{2 n+1}\right) .
$$

By continuity there exist two constants $x_{0}, t_{0}$ such that $v(x, 0)>0$ for $x \in\left(0, x_{0}\right)$, $v\left(x_{0}, t\right)>0$ for $t \in\left(0, t_{0}\right)$.

Now, if $v(0, t)$ were not positive for $0<t \leq t_{0}$, then it would assume a nonpositive minimum somewhere in the same interval. At that point, $v_{x}$ would also be nonpositive, thus contradicting Hopf's lemma.

REMARK 3.1. Condition (3.10) can be slightly generalized by replacing the constant 1 with a smooth function of $t, \bar{u}(t)$. In this case, one can easily see that the occurrence of a waiting time is guaranteed in the case $\bar{u}(t) \geq c>0$. 
4. Heat exchange with a well-stirred fluid. The mathematical formulation of this kind of boundary conditions (sometimes called a condition of fourth or fifth type; see e.g. [12, 13]) involves a new unknown function $V(t)$, the temperature of the wellstirred fluid, and the conditions to be imposed are:

a) conditions on the thermal contact between the medium under consideration and the well-stirred fluid,

b) energy balance in the well-stirred fluid.

Moreover, the initial value $V^{*}$ of $V(t)$ has to be given.

We refer to one-dimensional heat conduction in the cylinder $x>0$ with unitary cross section and isolated local surface, and we assume that the well-stirred fluid only exchanges energy across $x=0$. We have that condition a) can be stated either as a perfect thermal contact

$$
u(0, t)=V(t), t>0
$$

or as a boundary layer condition

$$
\frac{u(0, t)-V(t)}{\delta}=K u_{x}(0, t), t>0 .
$$

Considering condition b), we can write

$$
\frac{d E}{d t}=K u_{x}(0, t), \quad E(0)=E^{*}
$$

(both in (4.2) and (4.3) we will set $K=1$ consistently with the normalization we used in writing the heat conduction equation in the form (2.1)).

In (4.3), $E(t)$ is the thermal energy stored in the well-stirred fluid, and thus

$$
E(t)=C V(t),
$$

where $C$ is the thermal capacity of the fluid, possibly dependent on $V$.

If we are in the case of perfect thermal contact, so that

$$
u_{x}(0, t)=C \dot{V}, \quad u(0, t)=V(t), \quad V(0)=V^{*},
$$

the phenomenon of waiting time cannot take place even if $C$ has a jump for some $V=V_{0}$. Indeed, this would mean that $u(0, t)=V_{0}, u_{x}(0, t)=0$ over a finite time interval, implying

$$
\left.\frac{\partial^{m}}{\partial x^{m}} u(x, t)\right|_{x=0}=0, \text { for } m=1, \ldots
$$

and, by the analyticity of $u$, this is compatible only with $u(x, t) \equiv V_{0}$.

On the contrary, it is easily seen that in the case of imperfect thermal contact we can have waiting time.

For instance

$$
\left\{\begin{array}{l}
u_{x}(0, t)=C \dot{V}(t), \\
V(0)=V^{*}>0 \\
u(x, 0)=\phi(x)<0, x \geq 0, \\
u_{x}(0, t)=\frac{1}{\delta}[u(0, t)-V(t)]
\end{array}\right.
$$


can produce waiting time if

$$
\begin{aligned}
& \int_{0}^{+\infty} \phi(x) d x+C V^{*}>0, \\
& \frac{1}{\delta}= \begin{cases}A, & u<0, \\
A-B, & u>0, A, B>0 .\end{cases}
\end{aligned}
$$

Indeed $u(0, t)$ is forced to vanish for the first time at some instant $\hat{t}$ while $V(\hat{t})>0$ because of Hopf's lemma.

REMARK 4.1. Of course in the case of "very large" $C$, the "well-stirred fluid" becomes a thermostat and (4.1), (4.5) reduce to a constant Dirichlet datum whereas (4.2), (4.5) become a Robin-type condition.

Moreover, note that condition (4.3) can be generalized if we assume the presence of heat sources in the fluid.

5. Remarks on existence and uniqueness. We restrict ourselves to the problem described in section 2. For simplicity we consider as initial datum

$$
\Phi(x)=-A x+a x^{2}, \quad x>0,
$$

where $A$ and $a$ are positive constants.

We take a linear approximation of the Heaviside graph,

$$
H^{\varepsilon}= \begin{cases}0, & u \leq 0 \\ \frac{u}{\varepsilon}, & u \in[0, \varepsilon] \\ 1, & u \geq \varepsilon\end{cases}
$$

and, for any $\varepsilon \in(0,1)$, we solve the problem

$$
\begin{cases}u_{x x}^{\varepsilon}-u_{t}^{\varepsilon}=0, & x>0, t>0, \\ u^{\varepsilon}(x, 0)=\Phi(x), & x>0, \\ u_{x}^{\varepsilon}(0, t)=-A+H^{\varepsilon}\left(u^{\varepsilon}(0, t)\right) B, & t>0,\end{cases}
$$

and we note that $u_{t}^{\varepsilon}(0, t)$ is necessarily positive because of the maximum principle and Hopf's lemma.

As long as $u^{\varepsilon}(0, t)<\varepsilon$ we can write

$$
u^{\varepsilon}(x, t)=-A x+a x^{2}+2 a t+W(x, t),
$$

where $W$ solves a diffusion problem with vanishing initial datum and with boundary condition

Now we set

$$
W_{x}(0, t)-\frac{B}{\varepsilon} W(0, t)=\frac{2 B a t}{\varepsilon} .
$$

$$
z(x, t)=W_{x}(x, t)-\frac{B}{\epsilon} W(x, t),
$$

vanishing for $t=0$ and satisfying the boundary condition

$$
z(0, t)=\frac{2 B a t}{\varepsilon} .
$$


Elementary calculations yield

$$
z(x, t)=\frac{2 B a}{\varepsilon}\left\{\left(t+\frac{x^{2}}{2}\right) \operatorname{erfc} \frac{x}{2 \sqrt{t}}-x \sqrt{\frac{t}{\pi}} e^{-\frac{x^{2}}{4 t}}\right\} .
$$

We can integrate (5.6) (using the implicit condition that both $W$ and $z$ go to zero as $x \rightarrow+\infty)$ and obtain

$$
W(x, t)=-e^{-\frac{B}{\varepsilon} x} \int_{x}^{+\infty} e^{-\frac{B}{\varepsilon} \xi} z(\xi, t) d \xi
$$

implying in particular

$$
W(0, t)=-a \int_{0}^{+\infty}\left\{e^{-\eta}\left[2 t+\left(\frac{\varepsilon}{B} \eta\right)^{2}\right] \operatorname{erfc} \frac{\varepsilon \eta}{2 B \sqrt{t}}-\frac{\varepsilon \eta}{B} \sqrt{\frac{t}{\pi}} e^{-\frac{\varepsilon^{2} \eta^{2}}{4 B^{2} t}}\right\} d \eta
$$

After some manipulations we find

$$
u^{\varepsilon}(0, t)=\frac{4 a \sqrt{t}}{B \sqrt{\pi}} \varepsilon+o(\varepsilon), \text { as long as } u^{\varepsilon}<\varepsilon
$$

and, at first order in $\varepsilon$, we get that the time $t_{w}^{\varepsilon}$ such that $u^{\varepsilon}(0, x)=\varepsilon$ is given by

$$
\sqrt{t_{w}^{\varepsilon}}=\frac{B \sqrt{\pi}}{4 a}+o(\epsilon)
$$

in accordance with (2.18).

Obviously the above procedure can be repeated for any smooth approximation of the Heaviside graph.

More generally, we consider the problem

$$
\begin{cases}u_{t}=u_{x x}, & x>0, t>0, \\ u(x, 0)=\psi(x), & x>0, \\ u_{x}(0, t) \in G(u(0, t)), & t>0,\end{cases}
$$

where $G(u)$ is a piecewise continuous monotonically increasing graph for $u \in \mathbb{R}$. We define the classical solution to (5.13) in the usual way: $u \in C^{2,1}((0,+\infty) \times(0,+\infty))$, $u \in C([0,+\infty) \times[0,+\infty))$ with $u_{x}$ continuous up to $x=0$ (with appropriate growing conditions as $x \rightarrow \infty)$. We prove the following.

Theorem 5.1. Assume $\psi \in C^{2}[0,+\infty), \psi^{\prime}(0) \in G(\psi(0))$. Then (5.13) admits one unique solution and moreover

$$
\begin{aligned}
& u(x, t) \in \operatorname{Lip}([0,+\infty) \times[0,+\infty)), \\
& u_{x}(x, t) \in H^{1,1 / 2}([0,+\infty) \times[0,+\infty)),
\end{aligned}
$$

where $H^{1,1 / 2}$ denotes the space of functions that are Lipschitz-continuous w.r.t. $x$ and Hölder-continuous (with exponent $\frac{1}{2}$ ) w.r.t. $t$.

Proof. We start with a proof of existence. We consider a sequence of regularized problems

$$
\begin{cases}u_{t}^{\varepsilon}=u_{x x}^{\varepsilon}, & x>0, t>0 \\ u^{\varepsilon}(x, 0)=\psi(x), & x>0 \\ u_{x}^{\varepsilon}(0, t)=G^{\varepsilon}\left(u^{\varepsilon}(0, t)\right), & t>0\end{cases}
$$


where the regularized functions $G^{\varepsilon}(u)$ are continuously differentiable in $u$,

$$
\begin{aligned}
& \psi^{\prime}(0)=G^{\varepsilon}(\psi(0)), \\
& \lim _{\varepsilon \rightarrow 0} G^{\varepsilon}(u)=G(u) .
\end{aligned}
$$

Then the time derivative $v^{\varepsilon}=u_{t}^{\varepsilon}$ is a solution to the following problem:

$$
\begin{cases}v_{t}^{\varepsilon}=v_{x x}^{\varepsilon}, & x>0, t>0, \\ v^{\varepsilon}(x, 0)=\psi^{\prime \prime}(x), & x>0, \\ \frac{v_{x}^{\varepsilon}(0, t)}{v^{\varepsilon}(0, t)}=\frac{d G^{\varepsilon}\left(u^{\varepsilon}\right)}{d u^{\varepsilon}} \geq 0, & t>0 .\end{cases}
$$

Applying the maximum principle to the problem (5.19), we conclude that

$$
\left|v^{\varepsilon}(x, t)\right| \leq \max _{x \geq 0}\left|\psi^{\prime \prime}(x)\right| .
$$

This estimate yields

$$
\begin{aligned}
& u^{\varepsilon}(x, t) \in \operatorname{Lip}([0,+\infty) \times[0,+\infty)), \\
& u_{x}^{\varepsilon}(x, t) \in \operatorname{Lip}([0,+\infty)), \quad \forall t \geq 0 .
\end{aligned}
$$

Applying Lemma 3.1, ch. II of [11, we conclude that embedding (5.14), (5.15) holds for the sequence $u^{\varepsilon}$ uniformly in $\varepsilon>0$. Hence the proof of existence and of (5.14), (5.15) follows at once from a compactness argument.

The uniqueness result follows sraightforwardly from the following energy estimate:

$$
\int_{0}^{\infty}\left(u^{1}(x, t)-u^{2}(x, t)\right)^{2} d x \leq \int_{0}^{\infty}\left(\psi^{1}(x)-\psi^{2}(x)\right)^{2} d x
$$

where $u^{1}(x, t)$ and $u^{2}(x, t)$ are solutions to the problem (5.13) with initial values $\psi^{1}$ and $\psi^{2}$.

REMARK 5.2. Of course, assumptions of the previous theorem can be considerably weakened using estimates local in time. In this case, properties (5.14) and (5.15) are to be modified accordingly, requiring that they hold for any $[0,+\infty) \times[\delta,+\infty)$ and that the Lipschitz and Hölder constants are bounded by $C \delta^{-\gamma}$, for some positive $C$ and $\gamma$.

REMARK 5.3. An alternative procedure for studying the approximating sequence $u^{\varepsilon}$ could be the use of asymptotic matched expansions. It would have the advantage of providing some extra information on the structure of the transition from normal to waiting time regime and vice versa. We will not deal with this subject for the sake of brevity.

REMARK 5.4. If the multi-valued function $G$ is monotonic decreasing, waiting time does not occur (except in the trivial case described in the following remark). To show it in a simple example

$$
u_{x}(0, \tau)= \begin{cases}-A, & u<0, \\ -A-B, & u>0\end{cases}
$$

consider, as in section 2 the first $\tau_{0}$ such that $u\left(0, \tau_{0}\right)=0$ and take $n=1$ in (2.4) (it means that $\left.u_{\tau}\left(0, \tau_{0}\right)>0\right)$. The heat equation with $u_{0}(0, t)=-A-B$ and $u(x, 0)=$ $-A x+a x^{2}+o\left(x^{2}\right)$ is such that

$$
u(x, t)=-(A+B) x+v(x, t)
$$


when $v(x, t)$ has zero flux on $x=0$ and $v(x, 0)=+B x+o(x)$, and thus is positive.

On the other hand, imposing a waiting time we will have a boundary flux $-u_{x}(0, t)$ that becomes less than $A$.

REMARK 5.5. It is easily seen that the boundary condition (5.24) may produce nonuniqueness.

Consider again the heat equation in $\mathbb{R} \times \mathbb{R}$ and assume

$$
u(x, 0)=-A x .
$$

Of course $u(x, t)=-A x$ is a solution, but another solution is obtained by adding to $-A x$ the solution of a diffusion problem with zero initial datum and entering flux equal to $B$, which is locally positive on $x=0$.

6. Occurrence of waiting times in some free boundary problem. In this section we give two simple examples of free boundary problems in which waiting times may occur.

The first is a generalization of the well-known Green-Ampt solution [10 for the penetration of water in noncapillary soils, while the second is a diffusion problem with a threshold.

6.1. Imbibition of a filter. Consider a domain $\Omega:=\left\{(x, y, z): y^{2}+z^{2} \leq R^{2}, x>0\right\}$ occupied by a porous medium of porosity $\varepsilon$ and with negligible capillarity.

In the sequel we refer to the specific case in which the volume of the liquid occupying the unit volume of the porous medium $m(x, y, z, t)$ is such that

$$
m(x, y, z, t)= \begin{cases}0, & p(x, y, z, t) \leq 0, \\ \varepsilon, & p(x, y, z, t)>0,\end{cases}
$$

which corresponds to a porous medium without capillarity.

In (6.1), $p(x, y, z, t)$ is the pressure and $p=0$ the atmospheric pressure.

We consider a one-dimensional problem with no gravity and we assume

$$
p(x, 0)=0, x>0,
$$

i.e. the porous medium is initially dry.

We also assume that the boundary $x=0$ (which can be taken of unit cross section) is in contact with a reservoir in which the pressure is a prescribed positive function $p_{0}(t)$ of time and we suppose that the inflow discharge at $x=0$ is proportional to the pressure difference $p_{0}(t)-p(0, t)$. According to Darcy's law we can write:

$$
-p_{x}(0, t)=\alpha\left[p_{0}(t)-p(0, t)\right], t>0 .
$$

This corresponds to assuming that the flow from the reservoir has to cross a porous junction whose hydraulic resistivity is proportional to $\frac{1}{\alpha}$.

From the continuity equation we have

$$
p_{x x}=0, \text { where } p>0,
$$

i.e. in the water saturated region.

Thus the solution of our problem is given by: 
(i) a function $s(t)$ denoting the interface between the dry and wet porous medium $(s(0)=0$ because of $(\underline{6.2}))$,

(ii) a function $p(x, t)$ that depends linearly on $x \in(0, s(t))$.

Moreover on $x=s(t)$ we have

$$
\begin{aligned}
& p(s(t), t)=0, t>0, \\
& K p_{x}(s(t), t)=-\varepsilon \dot{s}(t), t>0,
\end{aligned}
$$

where the latter condition expresses the mass balance if $K$ is the hydraulic conductivity.

The situation just described is a simple generalization of the classical Green-Ampt model [10] in which the hydraulic contact between the porous medium and the reservoir is assumed to be perfect, so that

$$
p(0, t)=p_{0}(t)
$$

and thus

$$
\begin{aligned}
& s^{2}(t)=2 \frac{K}{\varepsilon} \int_{0}^{t} p_{0}(\tau) d \tau, \quad t>0 \\
& p(x, t)=p_{0}(t)\left(1-\frac{x}{s(t)}\right), \quad x \in(0, s(t)), \quad t>0
\end{aligned}
$$

(the classical Green-Ampt solution corresponds to $p_{0}=$ const.).

If the boundary condition is (6.3) we have, after obvious normalization, that $s(t)$ has to solve the O.D.E.

$$
\dot{s}+\alpha s \dot{s}=\alpha p_{0}(t)
$$

so that

$$
s(t)=\frac{-1+\sqrt{1+2 \alpha \pi(t)}}{\alpha}, \quad \dot{s}=\frac{\alpha p_{0}(t)}{\sqrt{1+2 \alpha \pi(t)}}
$$

and

$$
p(x, t)=\dot{s}(t)(s(t)-x), \quad x \in(0, s(t)), \quad t>0,
$$

where

$$
\pi(t)=\alpha \int_{0}^{t} p_{0}(\tau) d \tau
$$

Thus

$$
p(0, t)=-p_{0}(t)\left[1-\frac{1}{\sqrt{1+2 \alpha \pi(t)}}\right] .
$$

Now consider the case in which the junction "collapses" when $p(0, t)$ reaches some critical value $p^{*}$ and its resistivity jumps from the value $\frac{1}{\alpha}$ to the value $\frac{1}{\beta}>\frac{1}{\alpha}$ 는

If we assume that $p_{0}(t)$ is monotonic and that $p(0, t)$ crosses $p^{*}$ at some $t_{0}>0$, we can see that the free boundary has to stop for some finite waiting time.

Indeed, keeping a resistivity $\frac{1}{\alpha}$ after $t_{0}$ would lead to (see (‥14) $)$ a pressure $p(0, t)$ larger than $p^{*}$ (forcing the resistivity to $\frac{1}{\beta}$ ). On the contrary, taking $\frac{1}{\beta}$ after $t_{0}$ produces

\footnotetext{
${ }^{1}$ The limit case $\beta \rightarrow 0$ corresponds to the clogging of the junction.
} 
a discontinuity in the speed of the free boundary

$$
\dot{s}\left(t_{0}^{+}\right)-\dot{s}\left(t_{0}^{-}\right)=p_{0}\left(t_{0}\right)\left[\frac{\beta}{1+\beta s\left(t_{0}\right)}-\frac{\alpha}{1+\alpha s\left(t_{0}\right)}\right]=-\gamma<0,
$$

and thus $p\left(0, t_{0}^{+}\right)$would drop below $p^{*}$ :

$$
p\left(0, t_{0}^{+}\right)=p\left(0, t_{0}^{-}\right)+s\left(t_{0}\right)\left[\dot{s}\left(t_{0}^{+}\right)-\dot{s}\left(t_{0}^{-}\right)\right]=p^{*}-s\left(t_{0}\right) \gamma .
$$

We conclude that, starting from $t_{0}$ the condition to be imposed is

$$
p(0, t)=p^{*}, \quad t_{0}<t<t_{0}+\hat{t}
$$

and $\hat{t}$ is such that $-p_{x}(0, t)$ reaches the value $\beta\left(p_{0}(t)-p^{*}\right)$, i.e.

$$
\beta s\left(t_{0}+\hat{t}\right)=\alpha s\left(t_{0}\right) .
$$

After $t_{0}+\hat{t}$, the condition to be imposed is

$$
-p_{x}(0, t)=\beta\left(p_{0}(t)-p(0, t)\right), \quad t>t_{0}+\hat{t} .
$$

Therefore the correct form of the boundary condition holding for any $t>0$ is

$$
-p_{x}(0, t) \in\left\{\alpha+(\beta-\alpha) H\left(p(0, t)-p^{*}\right)\right\}\left(p_{0}(t)-p(0, t)\right) .
$$

6.2. Free boundary with Signorini type condition. Consider a substance that diffuses in a substrate following Fick's law, so that if $c(x, t)$ is the (normalized) concentration of the substance

$$
c_{x x}-c_{t}=0, \text { in the penetrated region, }
$$

where we are restricted to a one-dimensional problem, using directly nondimensional variables.

Penetration occurs if the concentration at the penetration front exceeds a threshold value $c^{*}$, and the penetration speed depends on the concentration on the front. This problem has been described e.g. in [2], 7].

Accordingly, if $x=s(t)$ denotes the location of the penetration front, we have that (6.21) holds, say, for $x \in(0, s(t)), t>0$ and

$$
\dot{s}(t)= \begin{cases}0, & \text { if } c(s(t), t)<c^{*}, \\ f(c(s(t), t)) & \text { if } c>c^{*} .\end{cases}
$$

In addition, conservation of mass at the free boundary reads

$$
-c_{x}(s(t), t)=\dot{s}(t) c(s(t), t) .
$$

In order to illustrate the occurrence of a waiting time we consider a model problem where $f \equiv 1$ and we assume that, at the time $t=0$ when the front concentration reaches the value $c^{*}$, the penetrated region is $x<0$.

Moreover we take

$$
c(x, 0)=c^{*}+a x^{2}, x<0=s(0),
$$

with $a>0$. If we apply (6.22), (6.23) with

$$
c_{x}(0, t)=0, t>0,
$$

we see that $c_{t}(0, t)=2 a>0$ for $t>0$, implying $c(0, t)>c^{*}$ and contradicting (6.22). 
On the other hand, consider the problem

$$
\begin{cases}c_{x x}-c_{t}=0, & x<t, t>0, \\ c(x, 0)=c^{*}+a x^{2}, & x<0, \\ c_{x}(t, t)+c(t, t)=0, & t>0 .\end{cases}
$$

Problem (6.26) is obtained by assuming that $c(s(t), t)>c^{*}$, and hence $\dot{s}(t)=1$ so that (6.23) is equivalent to the third condition.

If we show that $c(t, t)<c^{*}$ in some interval $(0, \tilde{t})$ it will be clear that $(6.22)$ is contradicted again and there will be some waiting time for the transition from the first condition in (6.22) to the second (with $f \equiv 1$ ).

For $\varepsilon>0$ and $\gamma>0$ to be determined, consider the function

$$
U_{\varepsilon}(x, t)=c^{*}+\varepsilon+a x^{2}-\gamma x+2 a t .
$$

We have

$$
\begin{gathered}
U_{\varepsilon}(x, 0)>c(x, 0), \\
U_{\varepsilon x}(t, t)+U_{\varepsilon}(t, t)=2 a t-\gamma+c^{*}+\varepsilon+a t^{2}-\gamma t+2 a t=: R_{\varepsilon}(t),
\end{gathered}
$$

and if $\gamma<c^{*}, R_{\varepsilon}(t)>0$ in $\left[0, t_{0}\right], \forall \varepsilon>0$.

This means (apply once again the boundary point principle)

$$
U_{\varepsilon}(t, t)>c(t, t) \text { in }\left[0, t_{0}\right] \forall \varepsilon .
$$

Consequently,

$$
c(t, t)<U_{0}(t, t)=c^{*}+a t^{2}-\gamma t+2 a t, \quad t \in\left[0, t_{0}\right]
$$

and if $\gamma>2 a$ we conclude that

$$
c(t, t)<c^{*}
$$

in some interval. Thus, by this simple argument we have produced an example $\left(c^{*}>2 a\right)$ in which waiting time occurs.

REMARK 6.1. Thus, again, the correct way of formulating condition (6.22) is

$$
\dot{s}(t) \in f(c(s(t), t)) H\left(c(s(t), t)-c^{*}\right),
$$

and note that during the waiting time, when we "freeze" $c(s(t), t)$ to $c^{*}$ the free boundary conditions become

$$
\left\{\begin{array}{l}
c(s(t), t)=c^{*}, \\
-c_{x}(s(t), t)=c^{*} \dot{s},
\end{array}\right.
$$

i.e. conditions of Stefan type.

REMARK 6.2. In all the examples presented in this paper the occurrence of a waiting time is motivated by the necessity of the considered system to enter a dynamical process, gradually modifying some of its physical properties until some particular condition is met, while a relevant quantity remains frozen at some critical value. Of course one could think of different types of problems in which some time has to elapse before some critical phenomenon takes place. Such a class of problems is not the same as the one we have been focussing on. Nevertheless, one could still talk of waiting time, but in a different spirit. A very typical example is provided by the hyperbolic and the parabolic obstacle problems, in which the waiting time is actually the flight time to the obstacle. The 
well-known vibrating string with an obstacle is quite clear. The parabolic analogue is represented for instance by the problem

$$
\begin{gathered}
u_{t}-u_{x x}=-1, \quad x>0, t>0, \\
u(x, 0)=0, \quad x>0, \\
u(0, t)=0, \quad t>0
\end{gathered}
$$

with the obstacle $u(x, t) \geq-A x, A>0$.

Before hitting the obstacle the solution is of the type $u(x, t)=t f\left(\frac{x}{\sqrt{t}}\right)$, with $f\left(\frac{x}{\sqrt{t}}\right)$ solving the o.d.e. $f^{\prime \prime}+\frac{1}{2} \xi f^{\prime}-f=1$, with $\xi=\frac{x}{\sqrt{t}}$, and $f(0)=0$.

The slope $u_{x}(0, t)$ is initially zero and it keeps decreasing until it reaches the value $-A$ at some time $t^{*}$, which is the flight time to the obstacle. What happens next is that a coincidence set $0<x<s(t)$ propagates. The problem becomes a free boundary problem with the conditions of Cauchy type:

$$
u(s(t), t)=-A s(t), \quad u_{x}(s(t), t)=-A,
$$

which is in turn reducible to a classical Stefan problem for the unknown $z=u_{t}$.

It is worth noting that obstacle problems of this kind arise in the Black-Scholes model in finance (see [14]) when considering American options with piecewise linear payoff.

Acknowledgments. The authors are grateful to Professor Sam Howison for his useful comments and suggestions.

\section{REFERENCES}

[1] D.G. Aronson, The porous media equation in "nonlinear diffusion problems", Springer Lecture Notes in Mathematics, no. 1224, 1986, pp. 1-46, A. Fasano, M. Primicerio, eds. MR 877986 (88a:35130)

[2] G. Astarita and G. Sarti, A class of mathematical models for sorption of swelling solvents in glassy polymers, Polym. Eng. Sci. 18 (1978), 388-395.

[3] G. I. Barenblatt, On some unsteady motions of a liquid and gas in a porous medium, Prikl. Math. Meh. 16 (1952), 67-78. MR0046217(13:700a)

[4] J.R. Cannon, The one-dimensional heat equation, Encyclopedia of Mathematics and its Applications, vol. 23, Addison-Wesley, Menlo Park, 1984. MR747979 (86b:35073)

[5] H.S. Carslaw and J.C. Jaeger, Conduction of heat in solids, Clarendon Press, Oxford, 1959. MR0022294 (9:188a)

[6] A. Fasano and A. Mancini, A phenomenon of waiting time in phase change problems driven by radiative heat transfer, Math. Meth. Appl. Sci. 32 (2009), 1105-1117. MR2523565

[7] A. Fasano, G.H. Meyer, and M. Primicerio, On a problem in the polymer industry: theoretical and numerical investigation of swelling, SIAM J. Math. Anal. 17 (1986), 945-960. MR846399 (87h:35355)

[8] A. Friedman, Partial differential equations of parabolic type, Prentice-Hall, Englewood Cliffs, 1964. MR0181836 (31:6062)

[9] M. Gevrey, Sur les équations aux dérivées partielles du type parabolique, J. de Math. 10 (1913), no. $6,105-148$.

[10] W.H. Green and G.A. Ampt, Studies in soil physics. The flow of air and water through soils, J. Agric. Sci. 4 (1911), 1-24.

[11] O.A. Ladyzenskaja, N.A. Solonnikov, and N.N. Uraltseva, Linear and quasi-linear equations of parabolic type, Transl. Math. Monogr., vol. 23, American Mathematical Society, Providence, 1968. 
[12] A. Mikelic and M. Primicerio, Homogenization of the heat equation for a domain with a network of pipes with a well-mixed fluid, Ann. Mat. Pura Appl. 166 (1994), no. 4, 227-251. MR1313806 (96f:35167)

[13] M. Ughi, Teoremi di esistenza per problemi al contorno di iv ev tipo per una equazione parabolica lineare, Riv. Mat. Univ. Parma 5 (1979), 591-606.

[14] P. Willmott, J.N. Dewynne, and S.D. Howison, Option pricing: Mathematical models and computation, Financial Press, Oxford, 1993. 